\title{
Neoadjuvant $N a b$-paclitaxel and Gemcitabine in Borderline Resectable or Locally Advanced Unresectable Pancreatic Adenocarcinoma in Patients Who Are Ineligible for FOLFIRINOX
}

\author{
SHAWN L. PETERSON ${ }^{1}$, MUHAMMAD HUSNAIN ${ }^{2}$, TERRI POLLACK ${ }^{2}$, AGUSTIN PIMENTEL $^{2}$, \\ ARTURO LOAIZA-BONILLA ${ }^{3}$, COLLEEN WESTENDORF-OVERLEY ${ }^{4}$, KELLEY RATERMANN $^{4}$, \\ LOWELL ANTHONY ${ }^{4}$, PHILIP DESIMONE ${ }^{4}$, GAURAV GOEL ${ }^{5}$, MAHESH KUDRIMOTI ${ }^{4}$, \\ SEAN DINEEN ${ }^{6}$, CHING-WEI D. TZENG $^{7}$ and PETER J. HOSEIN ${ }^{2}$ \\ ${ }^{1}$ University of Louisville, James Graham Brown Cancer Center, Louisville, KY, U.S.A.; \\ ${ }^{2}$ University of Miami, Sylvester Comprehensive Cancer Center, Miami, FL, U.S.A.; \\ ${ }^{3}$ Cancer Treatment Centers of America, Philadelphia, PA, U.S.A.; \\ ${ }^{4}$ University of Kentucky Markey Cancer Center, Lexington, KY, U.S.A.; \\ ${ }^{5}$ University of Pittsburgh, Hillman Cancer Center, Pittsburgh, PA, U.S.A.; \\ ${ }^{6}$ Moffitt Cancer Center, Tampa, FL, U.S.A.; \\ ${ }^{7}$ The University of Texas, MD Anderson Cancer Center, Houston, TX, U.S.A.
}

\begin{abstract}
Background/Aim: Combination nab-paclitaxel/ gemcitabine $(A G)$ is superior to gemcitabine in patients with metastatic pancreatic cancer $(P C)$. There are limited data for $A G$ in borderline resectable $(B R)$ or locally advanced pancreatic cancer ( $L A P C)$. Herein, we report our experience with neoadjuvant $A G$ for $B R / L A P C$ in patients ineligible for FOLFIRINOX. Patients and Methods: This retrospective series, included patients with $B R / L A P C$ who received $A G$ as neoadjuvant therapy for 3-4 months followed by radiation, then re-evaluation for surgery. Results: Between 10/20132/2018, 32 patients $(22 \mathrm{BR}, 10 \mathrm{LAPC})$ were treated with this approach. Median age was 70 years. Nine patients were converted to resectability by imaging; six had $R 0$ resections (19\%), five (16\%) achieved a partial response and 24 (75\%) had stable disease. Conclusion: In this small series, the RO resection rate and response rate were $19 \%$ and $16 \%$ respectively. These data suggest that neoadjuvant AG may be an alternate option for patients ineligible for FOLFIRINOX.
\end{abstract}

This article is freely accessible online.

Correspondence to: Peter J. Hosein, MD, 1475 NW 12th Ave. Suite 3400, Miami, FL, 33136, U.S.A. Tel: +1 3052433462, Fax: +1 3052434975, e-mail: phosein@miami.edu

Key Words: Pancreatic cancer, locally advanced, neoadjuvant, gemcitabine, $n a b$-paclitaxel.
Pancreatic ductal adenocarcinoma (PDAC) is the third leading cause of cancer deaths in the United States with a projected 44,330 deaths occurring in 2018 (1). With a 5-year overall survival (OS) rate of $8 \%$, it carries a bleak prognosis largely due to the advanced stage at presentation in most patients (1). Surgical resection in the context of multimodality therapy is the only treatment that carries a potential for cure but only $10-20 \%$ have anatomically resectable disease at presentation, and up to $50 \%$ of newly diagnosed patients present with non-metastatic unresectable disease. Based upon the extent of vascular involvement, these patients are defined as borderline resectable (BR) or locally advanced, pancreatic cancer (LAPC) (2). The main treatment goal for patients with initially unresectable pancreatic cancer is conversion to resectability with recent data showing that a margin-negative resection after neoadjuvant therapy is associated with better survival than those who did not have surgery $(2,3)$.

One of the most efficacious therapies for metastatic PDAC is the FOLFIRINOX regimen (5-fluorouracil, oxaliplatin, leucovorin and irinotecan). Conroy et al. demonstrated that FOLFIRINOX improved OS compared to gemcitabine, with a median OS of 11.1 months $v s .6 .8$ months in patients with metastatic disease (4). Patients were included in this study only if they were less than 75 years old and had an excellent performance status (Eastern Cooperative Oncology Group [ECOG] 0 or 1. Subsequent studies have shown that neoadjuvant FOLFIRINOX 
followed by combined chemoradiation therapy may be feasible as neoadjuvant therapy $(5,6)$. This algorithm can result in a microscopic margin-negative (R0) resection rate of approximately $18 \%$ in patients with initially LAPC, and higher for those who are $\operatorname{BR}(6,7)$. Von Hoff et al. have also shown an improved OS in patients with metastatic PDAC treated with gemcitabine plus nab-paclitaxel (AG) compared to gemcitabine alone with a median OS of 8.5 months compared to 6.7 months (7). This was one of the first trials to ever show a significant survival advantage for a gemcitabine-based doublet in the subgroup of patients with a Karnofsky performance status of 70-80 (equivalent to an ECOG performance status of 2). This regimen has also recently been used in the neoadjuvant setting for potentially resectable PDAC. Ielpo et al. demonstrated a $68 \%$ resection rate along with a median OS and disease-free survival of 31 months and 18 months respectively (8).

Following the phase III trial of $\mathrm{AG}$ in patients with advanced disease, we began using this regimen followed in some cases by chemoradiation in patients with BRPC or LAPC who were deemed ineligible for FOLFIRINOX neoadjuvant therapy due to age, poor performance status or comorbidities. Within this context, the aims of this retrospective study were to determine the $\mathrm{R} 0$ resection rate, response rate, progression free survival (PFS), and toxicity in this patient population.

\section{Patients and Methods}

A retrospective analysis was performed at the University of Kentucky Markey Cancer Center in Lexington, Kentucky and the University of Miami, Sylvester Cancer Center, Miami, FL of all patients with BRPC or LAPC who received first-line treatment with AG. Patients were identified by searching the pancreatic cancer database which was approved by the Institutional Review Board (IRB) of both institutions.

The medical, radiation and surgical oncologists in the group developed an algorithm for uniform treatment of this group of patients as follows. Patients were selected for treatment with AG if they had a histological or cytological diagnosis of pancreatic adenocarcinoma, an Eastern Cooperative Group performance status (ECOG PS) of 0-3, adequate organ function, BRPC or LAPC, or deemed ineligible for FOLFIRINOX neoadjuvant therapy. The decision about ineligibility for FOLFIRINOX was based on the judgement of the treating medical oncologist and usually was because of advanced age, poor performance status (PS) or significant medical comorbidities. For patients who presented with biliary obstruction, adequate biliary drainage was required prior to initiation of this chemotherapy. The radiographic determination of resectability was made by standardized multidisciplinary review. The NCCN (National Comprehensive Cancer Network) criteria for resectability were applied (9). The data used to determine resectability included pretreatment contrast-enhanced CT scans (CECT's) and endoscopic ultrasound (EUS) in all patients.

The treatment regimen was the same as published in the MPACT trial for metastatic PDAC (7). This consisted of gemcitabine $1,000 \mathrm{mg} / \mathrm{m}^{2}$ and $n a b$-paclitaxel $125 \mathrm{mg} / \mathrm{m}^{2}$ administered on days 1,8 and 15 every 28 days for at least 2-4 cycles. Treatment continued until progression of disease, intolerable toxicity or maximum response. Toxicities were assessed at every visit and graded according to the National Cancer Institute Common Terminology Criteria for Adverse Events, version 4. Criteria for dose modification were similar to those described in the MPACT trial. Response assessment by CECT's were performed every 8 weeks on therapy or sooner if progression was suspected by symptoms or rising serum CA19-9.

After every CECT scan during treatment, each patient's case was reviewed at a multidisciplinary conference to determine whether the reason for defining the patient as BRPC or UR LAPC had improved. Since size was not the only criterium used in this evaluation, traditional response criteria (such as RECIST) were not employed. If two consecutive scans during treatment showed similar findings with no improvement, this was considered to be the maximum response. Maximum tolerability was defined as the point when excessive toxicities warranted stopping AG, even if a patient had not achieved their maximum response. Because an algorithm of real-time monitoring of response and toxicity was used, there was no predefined minimum or maximum number of cycles.

At maximum response or maximum tolerability, patients who appeared to be resectable by imaging criteria were offered surgical exploration and resection (within 6-8 weeks after chemotherapy) followed by postoperative chemoradiation. Patients who remained UR at maximum response or tolerability of AG were offered chemoradiation. For radiation sensitization, patients received concurrent capecitabine. Intensity-modulated radiation therapy (IMRT) was delivered in a standard fashion to a total dose of 50.4 Gy in 28 fractions or $52.5 \mathrm{~Gy}$ in 25 fractions. At the end of chemoradiation, patients were re-evaluated with CECT's to determine resectability. Post-CCRT treatment was left to the discretion of the treating physicians. Dose intensity was defined as the percentage of the full dose that was delivered.

The primary endpoint for this analysis was R0 resection rate. Secondary endpoints included Overall survival (OS), progression free survival (PFS), response rate, and toxicity. An R0 resection was defined as at least $1 \mathrm{~mm}$ free margins. Patients known to be alive were censored at the time of last contact. All patients who received at least one cycle of AG were included in the analysis. PFS was defined as the duration in months from the date of the first cycle of AG until the date of documented progression, recurrence, or death, whichever was soonest. OS and PFS were estimated by the KaplanMeier method with corresponding two-sided $95 \%$ confidence interval (CI) for survival proportions based on Greenwood's variance and the $\log$-transform method. Statistical significance was defined as $p<0.05$, and all tests were two-sided. Tests were performed using the IBM SPSS statistics software version 24 (IBM, NY, USA).

\section{Results}

Between 10/2013-2/2018, 32 treatment-naïve BRPC or UR LAPC patients were treated with AG. Baseline characteristics are shown in Table I. The median age was 70 years (range $=44-90$ years). Fifty percent of the patients were deemed BR and $31 \%$ UR on initial diagnosis, $19 \%$ of the patients were resectable, but had other comorbidities (equivalent to the MD Anderson Cancer Center borderline resectable group C). ECOG PS ranged from 0-3 with $19 \%$ 
Table I. Baseline characteristics.

\begin{tabular}{lc}
\hline & $\mathrm{N}(\%)$ \\
\hline Age (median, range), years & $70(44-90)$ \\
Gender & $10(31 \%)$ \\
Male & $22(68 \%)$ \\
Female & $5(16 \%)$ \\
ECOG performance status & $21(66 \%)$ \\
0 & $4(13 \%)$ \\
1 & $2(6 \%)$ \\
2 & \\
3 & $10(31 \%)$ \\
Reason for neoadjuvant therapy & $16(50 \%)$ \\
Unresectable & $6(19 \%)$ \\
Borderline resectable & \\
Resectable but comorbidities & $21(66 \%)$ \\
Requiring biliary stent & $11(34 \%)$ \\
Yes & \\
No &
\end{tabular}

of patients having a PS of 2 or 3 . Forty-four percent of patients were administered between 6-9 doses of AG while $16 \%$ received less than 6 doses and $40 \%$ received greater than 10 doses. The median number of administered doses was 9. In total, 319 doses of AG were given to the patients, with $231(72 \%)$ at reduced doses. Reasons for dose reductions were similar to the published AG regimen $(6,7)$ along with worsening clinical status. The mean dose intensity for gemcitabine and $n a b$-paclitaxel was $78 \%$ and $76 \%$ respectively.

Treatment outcomes are summarized in Table II. Prior to radiation therapy, the best radiological response was a partial response based on RECIST criteria in 5/32 (16\%) of patients; $75 \%(24 / 32)$ of patients had stable disease while $9 \%(3 / 32)$ had progression of disease prior to radiation. Sixty-one percent of patients received neoadjuvant chemoradiation. Overall six (19\%) patients achieved an R0 resection after neoadjuvant treatment and surgical exploration. Out of these six patients four had BRPC. Three patients were deemed unresectable at surgical exploration, one due to peritoneal metastases, one due to liver metastases, and the other was due to a grossly abnormal liver and biopsy showed necrotizing cholangitis of the right posterior section. Three patients were deemed to be surgical candidates after neoadjuvant therapy but declined surgery after discussing the risks and benefits. These patients were elderly, had complications during neoadjuvant therapy, and had concerns about postoperative quality of life. Three patients were considered medically unfit for surgery. Fourteen patients $(44 \%)$ had disease progression during or on the first scan after the neoadjuvant chemotherapy with or without chemoradiation program. Three patients are still receiving
Table II. Treatment outcomes.

\begin{tabular}{lc}
\hline Best radiological response to AG (prior to radiation) & $\mathrm{N}(\%)$ \\
\hline Partial response & $5(16 \%)$ \\
Stable disease & $24(75 \%)$ \\
Progression & $3(9 \%)$ \\
Radiation therapy received & $18(56 \%)$ \\
Surgery & \\
R0 resection & $6(19 \%)$ \\
Unresectable on exploration & $3(9 \%)$ \\
Refused surgery & $3(9 \%)$ \\
Medically unfit for surgery & $3(13 \%)$ \\
Disease progression on neoadjuvant therapy & $14(44 \%)$ \\
Ongoing response pending radiation therapy and or surgery & $3(9 \%)$ \\
\hline
\end{tabular}

AG: $N a b$-paclitaxel/gemcitabine.

neoadjuvant chemotherapy with good ongoing response with plan to proceed to surgery with or without radiation.

The median overall survival (OS) was 13.1 (95\%CI=9.217.0) months for all 32 treated patients. Median OS for 6 resectable patients (BR type $\mathrm{C}$ ) was not reached at the time of this analysis while it was 12 months $(95 \% \mathrm{CI}=8.4-15.6)$ for BR and 10.4 months $(95 \% \mathrm{CI}=3.3-16.8)$ for UR patients. PFS for all 32 treated patients was 8.8 months $(95 \% \mathrm{CI}=5.4-$ 12.2). Median PFS for 6 resectable patients was not reached at the time of this analysis and PFS for BR and UR was 8.4 $(95 \% \mathrm{CI}=5.5-11.3)$ and $7.4 \quad(95 \% \mathrm{CI}=2.4-12.1)$ months respectively (Table III).

\section{Discussion}

Systemic therapy for advanced PDAC has improved over the last 5 years with the FOLFIRINOX and AG regimens both showing superiority over gemcitabine monotherapy $(3,6)$. The systemic treatment of BRPC and LAPC is evolving as the newer regimens are being applied to these patients who have potential consolidative local treatment options after neoadjuvant therapy. Although prospective phase III studies are ongoing, none has been reported yet to confirm the role of FOLFIRNOX or AG in BRPC and UR LAPC. Recently Hammel et al. presented the data from a phase II LAPACT trial of AG for patients with UR LAPC with an R0 resection rate of $7 \%$ and a median PFS of 10.2 months (10). Treatment guidelines in the US have now incorporated chemotherapy with FOLFIRINOX or AG as options for patients with nonmetastatic disease $(5,6,11)$. Due to concerns about the tolerability of FOLFIRINOX, it is usually only applied to younger patients with good performance status (9).

Herein we report results of the AG regimen in BRPC and UR LAPC patients deemed ineligible for FOLFIRINOX neoadjuvant therapy. In this small series, the R0 resection 
Table III. Survival results.

\begin{tabular}{|c|c|c|c|c|}
\hline & $\mathrm{N}$ & Median (months) & 95\% CI (months) & $p$-Value \\
\hline OS, all patients & 32 & 13.1 & $9.2-17.0$ & \\
\hline \multicolumn{5}{|l|}{ OS } \\
\hline Resectable with comorbidities & 6 & Not reached & & \\
\hline Borderline resectable & 16 & 12.0 & $8.4-15.6$ & \\
\hline Unresectable & 10 & 10.4 & $3.3-16.8$ & 0.201 \\
\hline \multicolumn{5}{|l|}{ OS } \\
\hline ECOG 0 & 5 & Not reached & & \\
\hline ECOG 1 & 21 & 13.8 & $9.0-18.6$ & \\
\hline ECOG 2-3 & 6 & 10.4 & $0.5-20.3$ & 0.335 \\
\hline \multicolumn{5}{|l|}{ OS } \\
\hline No radiation & 14 & 6.4 & Not calculable & \\
\hline Radiation & 18 & 13.1 & $10.3-15.9$ & 0.702 \\
\hline PFS, all patients & 32 & 8.8 & $5.4-12.2$ & \\
\hline \multicolumn{5}{|l|}{ PFS } \\
\hline Resectable with comorbidities & 6 & Not reached & & \\
\hline Borderline resectable & 16 & 8.4 & $5.5-11.3$ & \\
\hline Unresectable & 10 & 7.4 & $2.4-12.1$ & 0.111 \\
\hline \multicolumn{5}{|l|}{ PFS } \\
\hline ECOG 0 & 5 & Not reached & & \\
\hline ECOG 1 & 21 & 8.8 & $5.7-11.9$ & \\
\hline ECOG 2-3 & 6 & 7.4 & $4.0-10.8$ & 0.369 \\
\hline \multicolumn{5}{|l|}{ PFS } \\
\hline No radiation & 14 & 5.8 & Not calculable & \\
\hline Radiation & 18 & 8.8 & $6.2-11.5$ & 0.990 \\
\hline
\end{tabular}

CI: Confidence interval; ECOG: Eastern Cooperative Oncology Group; OS: overall survival; PFS: progression-free survival.

rate was $19 \%$ and the response rate was at least $16 \%$, despite frequent dose reductions and relatively low dose intensity. As a comparison, a recent study of highly selected, good performance status UR/BRPC patients treated with neoadjuvant FOLFIRINOX followed by radiation also achieved an R0 resection rate of $20 \%(6,12)$. Although FOLFIRINOX demonstrates superior efficacy over gemcitabine in metastatic PDAC, there are still concerns about its tolerability, leading many investigators to modify the treatment regimen $(9,11)$. In our cohort, $72 \%$ of $A G$ doses had to be reduced, but no patient was taken off chemotherapy due to intolerance despite having patients with ECOG PS up to 3 and ages up to 90.

Historically, elderly and/or poor PS patients with PDAC, both of whom represent a clinically significant proportion of real-world patients outside of clinical trial populations, have been excluded from curative-intent strategies (13). Our data suggest that these patients may now have a possibility for potentially curative resection with the use of neoadjuvant AG.

\section{References}

1 Siegel RL, Miller KD and Jemal A: Cancer statistics, 2018. CA Cancer J Clin 68(1): 7-30, 2018.
2 Denbo JW and Fleming JB: Definition and Management of Borderline Resectable Pancreatic Cancer. Surg Clin North Am 96(6): 1337-1350, 2016.

3 Hosein PJ, Macintyre J, Kawamura C, Maldonado JC, Ernani V, Loaiza-Bonilla A, Narayanan G, Ribeiro A, Portelance L, Merchan JR, Levi JU and Rocha-Lima CM: A retrospective study of neoadjuvant FOLFIRINOX in unresectable or borderlineresectable locally advanced pancreatic adenocarcinoma. BMC Cancer 12: 199, 2012.

4 Conroy T, Desseigne F, Ychou M, Bouche O, Guimbaud R, Becouarn Y, Adenis A, Raoul JL, Gourgou-Bourgade S, de la Fouchardière C, Bennouna $\mathrm{J}$, Bachet JB, Khemissa-Akouz F, Péré-Vergé D, Delbaldo C, Assenat E, Chauffert B, Michel P, Montoto-Grillot C, Ducreux M, Groupe Tumeurs Digestives of Unicancer and PRODIGE Intergroup: FOLFIRINOX versus gemcitabine for metastatic pancreatic cancer. N Engl J Med 364(19): 1817-1825, 2011.

5 Yoo C, Kang J, Kim KP, Lee JL, Ryoo BY, Chang HM, Lee SS, Park DH, Song TJ, Seo DW, Lee SK, Kim MH, Park JH, Hwang DW, Song KB, Lee JH and Kim SC4: Efficacy and safety of neoadjuvant FOLFIRINOX for borderline resectable pancreatic adenocarcinoma: improved efficacy compared with gemcitabine-based regimen. Oncotarget 8(28): 46337-46347, 2017.

6 Suker M, Beumer BR, Sadot E, Marthey L, Faris JE, Mellon EA, El-Rayes BF, Wang-Gillam A, Lacy J, Hosein PJ, Moorcraft SY, Conroy T, Hohla F, Allen P, Taieb J, Hong TS, Shridhar R, 
Chau I, van Eijck CH and Koerkamp BG: FOLFIRINOX for locally advanced pancreatic cancer: a systematic review and patient-level meta-analysis. Lancet Oncol 17(6): 801-810, 2016.

7 Von Hoff DD, Ervin T, Arena FP, Chiorean EG, Infante J, Moore M, Seay T, Tjulandin SA, Ma WW, Saleh MN, Harris M, Reni M, Dowden S, Laheru D, Bahary N, Ramanathan RK, Tabernero J, Hidalgo M, Goldstein D, Van Cutsem R, Wei X, Iglesias J and Renschler MF: Increased survival in pancreatic cancer with $n a b$ paclitaxel plus gemcitabine. N Engl J Med 369(18): 1691-1703, 2013.

8 Ielpo B, Duran H, Diaz E, Fabra I, Caruso R, Ferri V, Malavé L, Hidalgo M, Alvarez R, Plaza C, Quijano Y and Vicente E2: Preoperative treatment with gemcitabine plus $n a b$-paclitaxel is a safe and effective chemotherapy for pancreatic adenocarcinoma. Eur J Surg Oncol 42(9): 1394-1400, 2016.

9 Tempero MA, Malafa MP, Al-Hawary M, Asbun H, Bain A, Behrman SW, Benson AB 3rd, Binder E, Cardin DB, Cha C, Chiorean EG, Chung V, Czito B, Dillhoff M, Dotan E, Ferrone CR, Hardacre J, Hawkins WG, Herman J, Ko AH, Komanduri S, Koong A, LoConte N, Lowy AM, Moravek C, Nakakura EK, O'Reilly EM, Obando J, Reddy S, Scaife C, Thayer S, Weekes CD, Wolff RA, Wolpin BM, Burns J and Darlow S: Pancreatic adenocarcinoma, version 2.2017, NCCN Clinical Practice Guidelines in Oncology. J Natl Compr Canc Netw 15(8): 10281061, 2017.
10 Hammel P, Lacy J, Portales F, Sobrero AF, Pazo Cid RA, Manzano Mozo JL, Terrebonne E, Dowden SD, Li JS, Ong TJ, Nydam $T$ and Philip PA: Phase II LAPACT trial of $n a b-$ paclitaxel (nab-P) plus gemcitabine $(\mathrm{G})$ for patients with locally advanced pancreatic cancer (LAPC). J Clin Oncol 36(4 suppl): 204-204, 2018.

11 Kim R: FOLFIRINOX: a new standard treatment for advanced pancreatic cancer? Lancet Oncol 12(1): 8-9, 2011.

12 Khushman M, Dempsey N, Maldonado JC, Loaiza-Bonilla A, Velez M, Carcas L, Dammrich D, Hurtado-Cordovi J, Parajuli R, Pollack T, Harwood AP, Macintyre J, Tzeng CW, Merchan JR, Restrepo MH, Akunyili II, Ribeiro A, Narayanan G, Portelance L, Sleeman D, Levi JU, Lima CM and Hosein PJ: Full dose neoadjuvant FOLFIRINOX is associated with prolonged survival in patients with locally advanced pancreatic adenocarcinoma. Pancreatology 15(6): 667-673, 2015.

13 Li D, Capanu M, Yu KH, Lowery MA, Kelsen DP and O'Reilly EM: Treatment, Outcomes, and Clinical Trial Participation in Elderly Patients With Metastatic Pancreas Adenocarcinoma. Clin Colorectal Cancer 14(4): 269-76.e1, 2015.

Received May 14, 2018

Revised June 7, 2018

Accepted June 8, 2018 